\title{
Two-Dimensional Effects on the Oxygen Reduction Reaction and Irreversible Surface Oxidation of Metallic Ru Nanosheets and Nanoparticles
}

Daisuke Takimoto ${ }^{1, *}$, Wataru Sugimoto ${ }^{1,2, *}$, Qiuyi Yuan ${ }^{3}$, Naoki Takao ${ }^{3}$, Takanori Itoh $^{3}$, Truong Vinh Truong Duy ${ }^{3}$, Tsukuru Ohwaki ${ }^{3}$, Hideto Imai ${ }^{3, *}$

${ }^{1}$ Research Initiative for Supra-Materials (RISM), Shinshu University, 3-15-1 Tokida, Ueda, Nagano 386-8567, Japan

${ }^{2}$ Faculty of Textile Science and Technology, Shinshu University, 3-15-1 Tokida, Ueda, Nagano 386-8567, Japan

3 Device-functional analysis department, NISSAN ARC LTD., 1 Natsushima, Yokosuka, Kanagawa 237-0061, Japan

*Corresponding authors (equally contributed): daitaki@shinshu-u.ac.jp,wsugi@shinshuu.ac.jp, imai@nissan-arc.co.jp

Table of contents:

$>$ Supplementary Figures S1-S7

$>$ Supplementary Table S1 


\section{$\underline{\text { Supplementary Figures }}$}

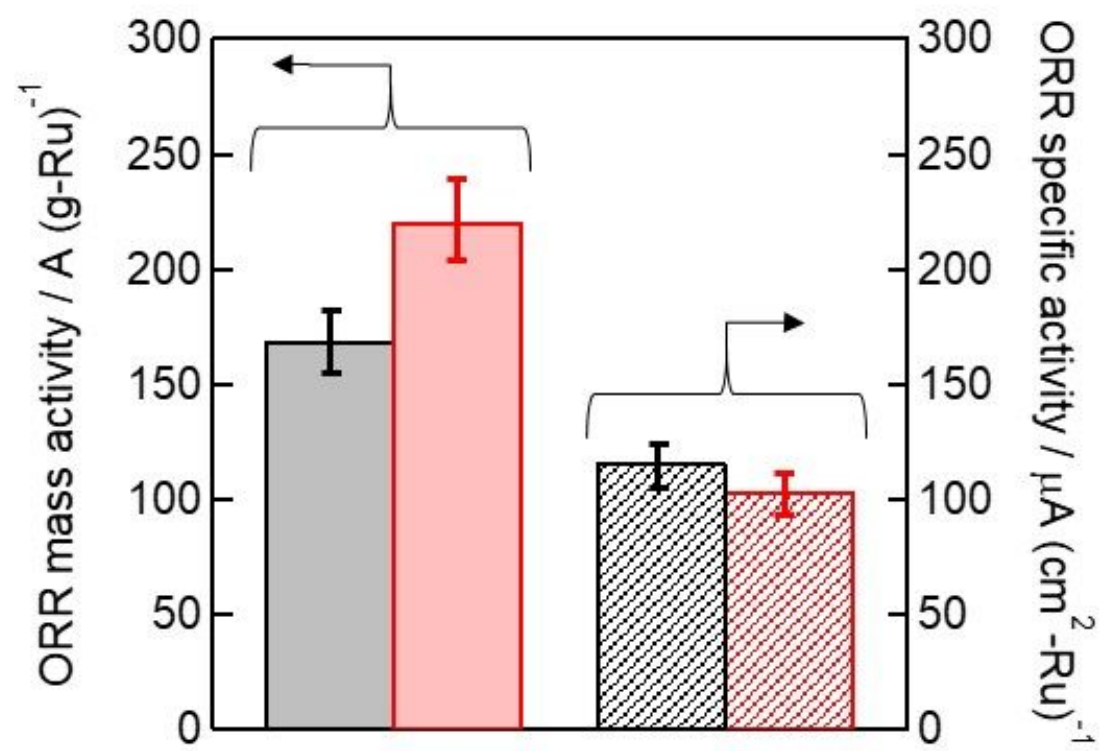

Figure S1. ORR mass and specific activity of fresh $\mathrm{Ru}(\mathrm{np}) / \mathrm{C}$ (black) and $\mathrm{Ru}(\mathrm{ns}) / \mathrm{C}$ (red). 


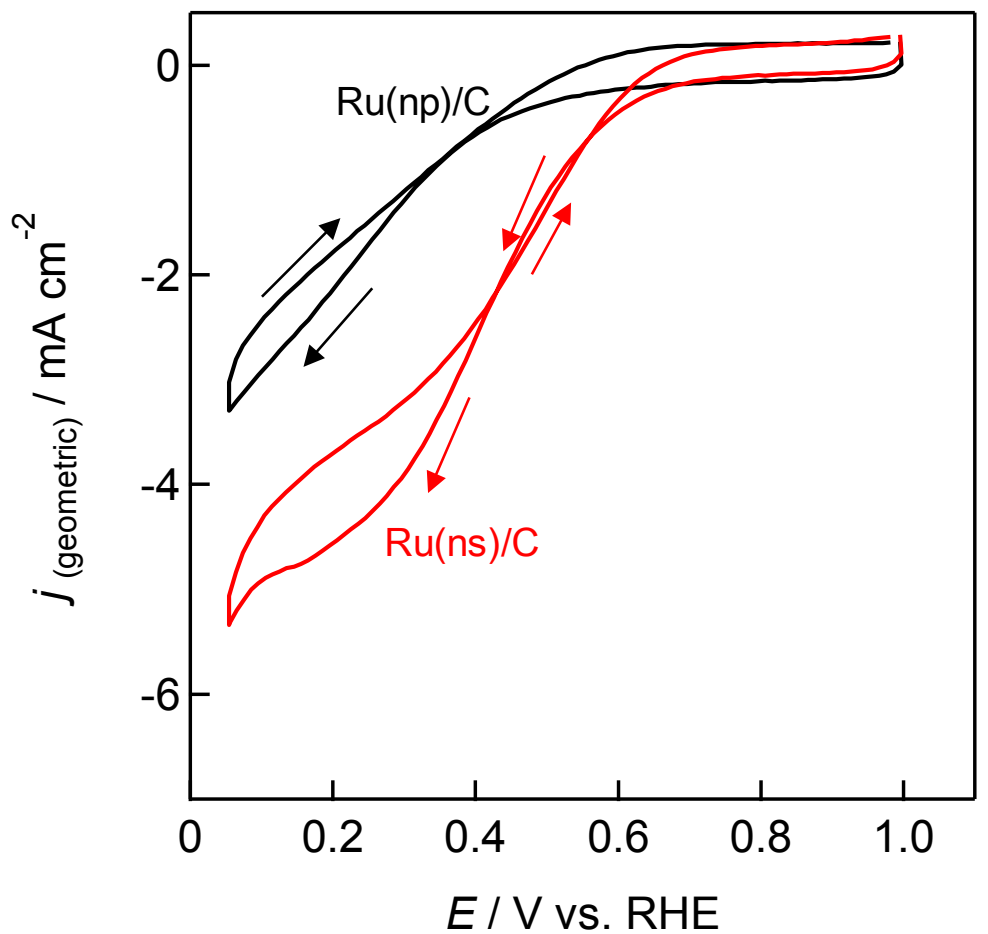

Figure S2. Cyclic voltammograms after 500 potential cycles of $\mathrm{Ru}(\mathrm{ns}) / \mathrm{C}$ (red) and $\mathrm{Ru}(\mathrm{np}) / \mathrm{C}$ (black) in $\mathrm{O}_{2}$-satureated $0.1 \mathrm{M} \mathrm{HClO}_{4}\left(60^{\circ} \mathrm{C}\right)$ at a scan rate of $10 \mathrm{mV} \mathrm{s}^{-1}$ and rotation rate of $1600 \mathrm{rpm}$. 
(a) Ru(ns)/C: before potential cycle

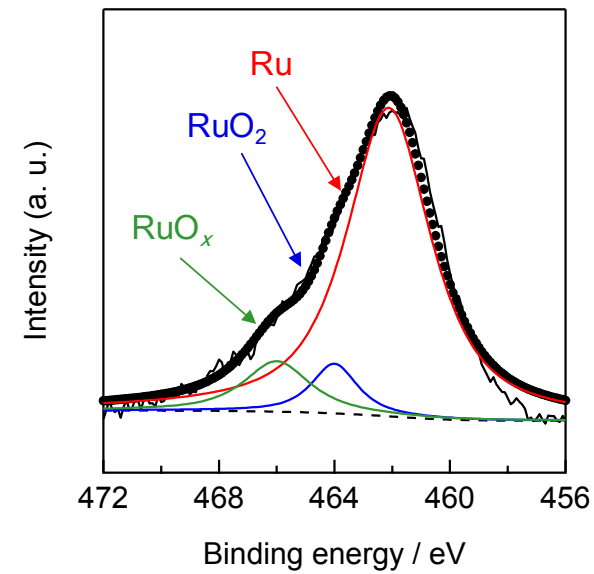

(c) Ru(ns)/C: after 500 cycles

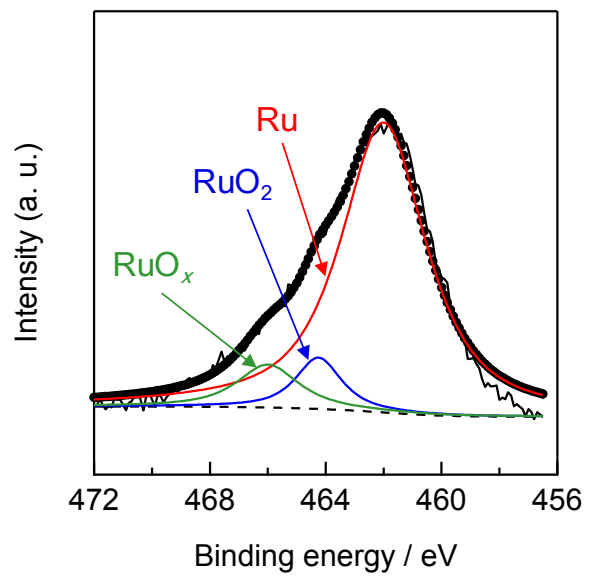

(e)

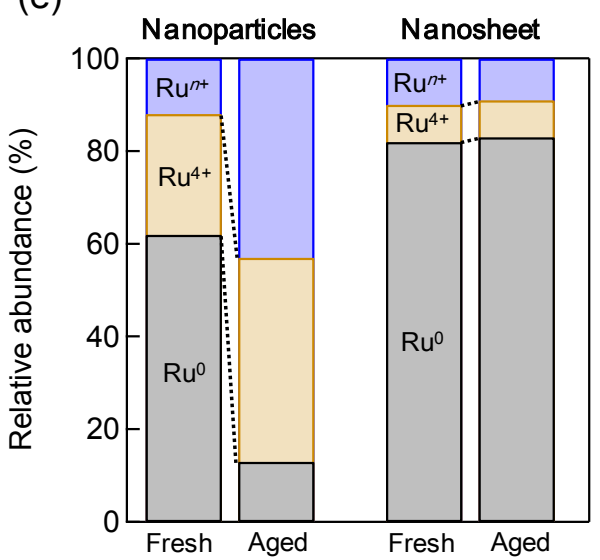

(b) Ru(np)/C: before potential cycle

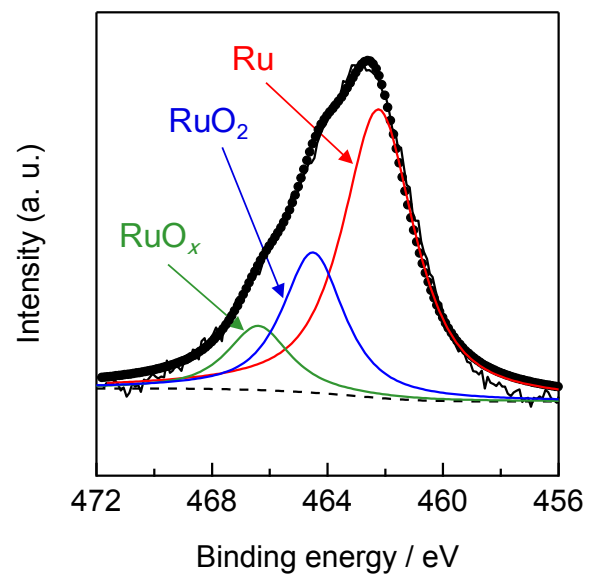

(d) Ru(np)/C: after 500 cycles

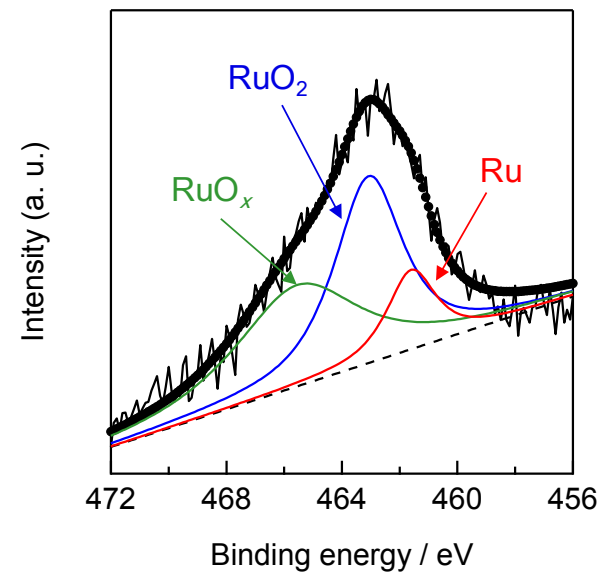

Figure S3. XPS spectrum of Ru3p $p_{3 / 2}$ for (a) $\mathrm{Ru}(\mathrm{ns}) / \mathrm{C}$ before potential cycle, (b) $\mathrm{Ru}(\mathrm{np}) / \mathrm{C}$ before potential cycle, (c) $\mathrm{Ru}(\mathrm{ns}) / \mathrm{C}$ after 500 potential cycles, (d) $\mathrm{Ru}(\mathrm{np}) / \mathrm{C}$ after 500 potential cycles. Red, blue and green lines indicate the $\mathrm{Ru}, \mathrm{RuO}_{2}$, and $\mathrm{RuO}_{x} \mathrm{H}_{y}$, respectively $(n>4)$. (e) Relative abundant of $\mathrm{Ru}$ species $\left(\mathrm{Ru}^{0}, \mathrm{Ru}^{4+}\right.$ and $\left.\mathrm{Ru}^{n+}(n>4)\right)$ obtained by curve fitting of XPS spectra for fresh and aged (after potential cycles) samples. 


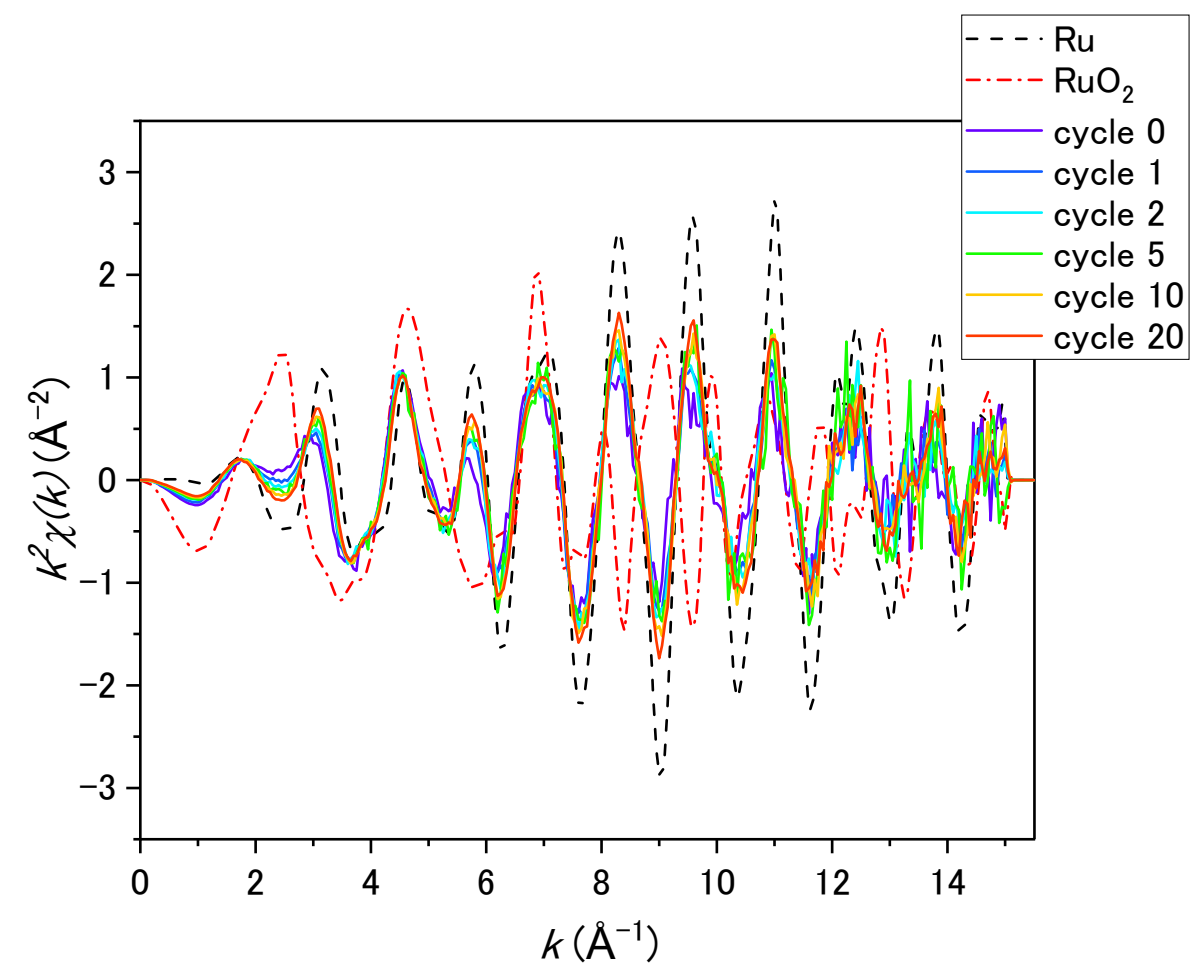

Figure S4. All Ru(ns)/C spectra presented similar oscillation period as Ru metal (black dots), but with smaller amplitude, indicating that the main interaction within $\mathrm{Ru}(\mathrm{ns}) / \mathrm{C}$ was $\mathrm{Ru}-\mathrm{Ru}$, and possibly with smaller CN/higher DW factor. 
a)

$$
\text { Top view }
$$

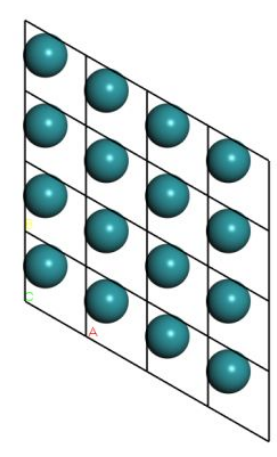

b)

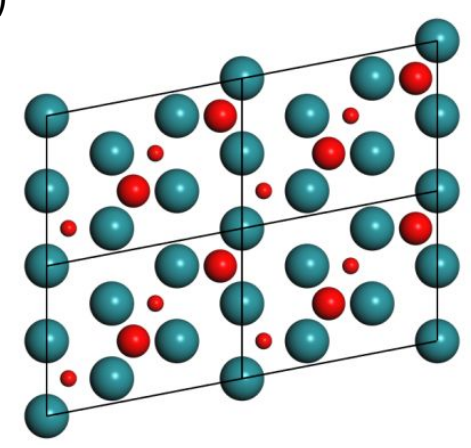

c)

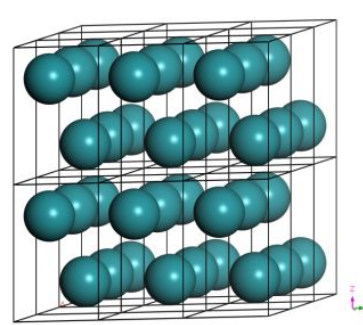

Side view

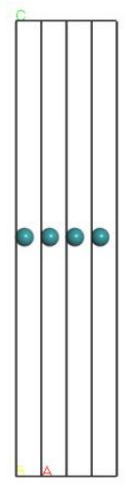

1-layer

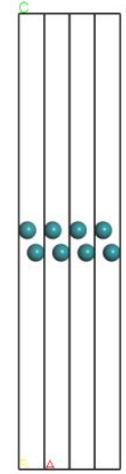

2-layer

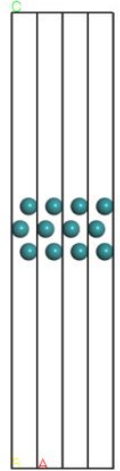

3-layer

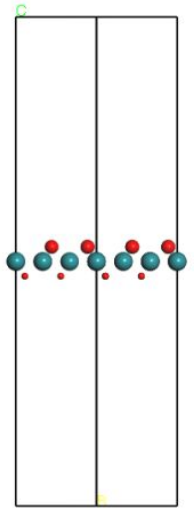

Figure S5. (a) Ru(ns) with 1-layer, 2-layer and 3-layer structure, (b) $\mathrm{O}_{\mathrm{ad}}$ on $\mathrm{Ru}(\mathrm{ns})$, and (c) $\mathrm{Ru}$ bulk. Blue: Ru, red: oxygen 

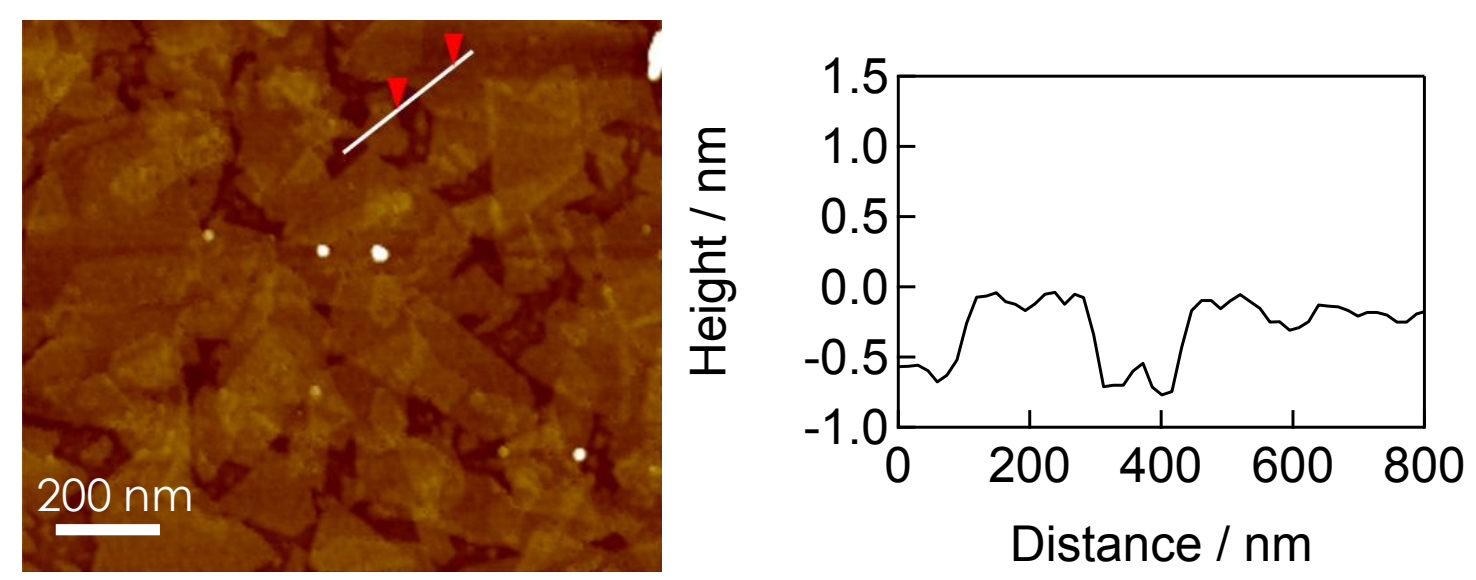

Figure S6. The average thickness of Ru nanosheets was $\sim 0.6 \mathrm{~nm}$. Since a monolayer $\mathrm{Ru}$ nanosheets has a thickness of $0.3 \mathrm{~nm}$, the thickness of $0.6 \mathrm{~nm}$ corresponds to a nanosheet with water molecule on the surface. 


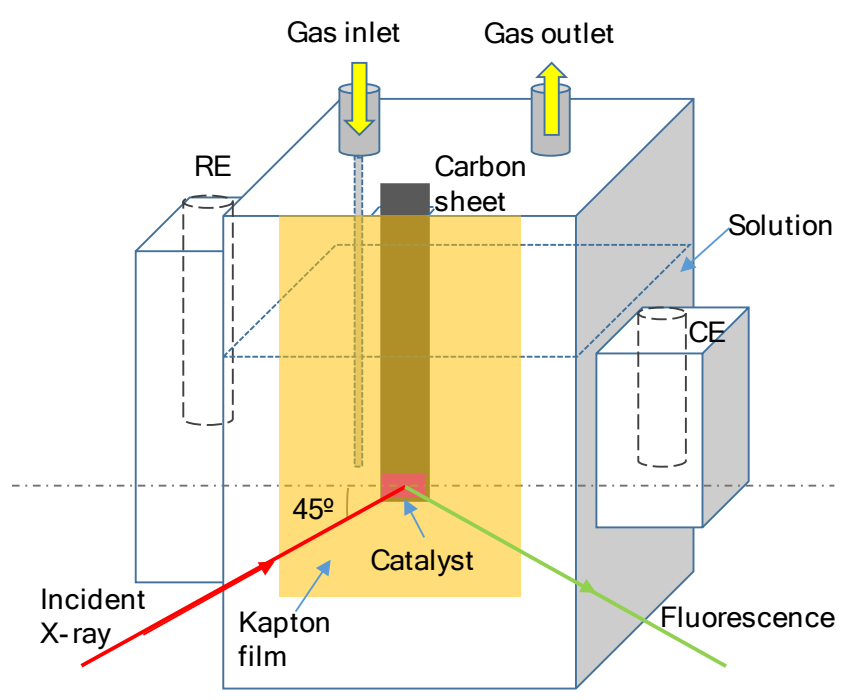

Figure S7. Schematic illustration of in-situ electrochemical XAS cell. 


\section{$\underline{\text { Supplementary Table }}$}

Table S1. XPS analysis results obtained by curve fitting of data shown in Fig. S2.

\begin{tabular}{llll}
\hline & Binding energy / eV & Assignment & Atomic ratio (\%) \\
\hline $\mathrm{Ru}(\mathrm{ns}) / \mathrm{C}$ before potential cycle & 462.1 & $\mathrm{Ru}^{0}$ & 82 \\
& 463.9 & $\mathrm{Ru}^{4+}$ & 7 \\
& 466.1 & $\mathrm{Ru}^{n}$ & 11 \\
$\mathrm{Ru}(\mathrm{np}) / \mathrm{C}$ before potential cycle & 462.6 & $\mathrm{Ru}^{0}$ & 62 \\
& 464.5 & $\mathrm{Ru}^{4+}$ & 26 \\
& 466.4 & $\mathrm{Ru}^{n}$ & 12 \\
$\mathrm{Ru}(\mathrm{ns}) / \mathrm{C}$ after potential cycle & 462.0 & $\mathrm{Ru}^{0}$ & 83 \\
& 464.3 & $\mathrm{Ru}^{4+}$ & 8 \\
$\mathrm{Ru}(\mathrm{np}) / \mathrm{C}$ after potential cycle & 461.5 & $\mathrm{Ru}^{n}$ & 9 \\
& 463.2 & $\mathrm{Ru}^{0}$ & 13 \\
& 465.3 & $\mathrm{Ru}^{4+}$ & 44 \\
\hline
\end{tabular}

